\title{
Histerectomia Laparoscópica em um Hospital Geral Comunitário Experiência Inicial e Comparação de Custos Hospitalares
}

\author{
Laparoscopic Hysterectomy in a Community General Hospital \\ Initial Experience and Comparison of Hospital Costs
}

\begin{abstract}
Randal Henrique de Oliveira, Antônio Alberto Nogueira, Álvaro Augusto Trigo, Francisco Spanó Neto, João Davanço Neto, Elias Dias Martins Jr., Luciano Costa Marques
\end{abstract}

\section{RESUMO}

Objetivo: comparar os custos hospitalares entre a histerectomia vaginal assistida por laparoscopia (HVAL) e a histerectomia total abdominal (HTA), relatando a experiência inicial com a nova abordagem em um hospital geral comunitário.

Pacientes e Métodos: foram comparados 11 casos de HVAL e 23 de HTA, realizados de setembro de 1998 a julho de 1999. Prontuários e demonstrativos das despesas hospitalares de cada paciente foram revistos para coletar as variáveis analisadas.

Resultados: não houve diferença estatística entre os grupos quanto à idade, paridade $e$ cirurgia abdominal prévia. A principal indicação cirúrgica para ambos os grupos foi leiomiomatose uterina. O grupo das HVAL apresentou tempo de internação menor, com mediana de 1 dia e o das HTA, de 2 dias (p<0,01). As HVAL mostraram-se 40,2\% mais caras que as HTA ( $p<0,01)$. Os gastos de centro cirúrgico contribuíram com a maior parte dos custos hospitalares para ambos os grupos, correspondendo a 79,8 e 57,9\% do total para as HVAL $e$ HTA, respectivamente. Os gastos de enfermaria para as HVAL foram menores que para as HTA, com diferença estatisticamente significativa ( $p=0,002)$.

Conclusão: com menor tempo de internação e com custos de enfermaria menores demonstramos que a HVAL propicia melhores condições de pós-operatório e recuperação mais rápida que a HTA. A HVAL, quando executada em um hospital geral comunitário, apesar de mais cara que a HTA, é uma ótima opção para a remoção uterina, e deve fazer parte do arsenal terapêutico dos cirurgiões ginecológicos.

PALAVRAS-CHAVE: Histerectomia. Laparoscopia. Custos em saúde.

\section{Introdução}

Em 1989 Reich et al. ${ }^{1}$ descreveram a primeira histerectomia com auxílio de técnicas laparoscópicas. Vários estudos têm demonstrado vantagens na abordagem laparoscópica para o tratamento cirúrgico de diferentes afecções em ginecologia ${ }^{2}$, porém ampla discussão vem sendo levantada quanto aos beneficios e a real utilidade desta abordagem para a remoção uterina ${ }^{3,4}$.

Setor de Ginecologia e Obstetrícia da Santa Casa de Misericórdia de Monte Alto

Correspondência:

Randal H Oliveira

Rua Antônio da Silva, 256

15910-000 - Monte Alto - SP

e-mail:randal@montealto.net
A histerectomia vaginal assistida por laparoscopia (HVAL) estaria indicada em mulheres com contra-indicações relativas à histerectomia vaginal (algia pélvica crônica, endometriose, antecedentes de cirurgia pélvica, existência de massa anexial, necessidade de ooforectomia e acesso vaginal dificil) e nas quais uma histerectomia total abdominal (HTA) seria habitualmente realizada. A nova abordagem conta com vantagens conhecidas para a via vaginal: menor morbidade intra-operatória, menor tempo de internação e menor tempo de convalescência. A via laparoscópica combinada com o procedimento vaginal permite ao cirurgião desfazer aderências com segurança, abordar focos de endometriose, avaliar panoramicamente a pelve e o abdome, realizar coleta de lavado peritoneal e facilmente remover 
os anexos uterinos, quando indicado ${ }^{5,6}$.

Em relação aos custos, porém, há estudos que criticam os gastos elevados com as técnicas laparoscópicas ${ }^{7,8}$, ao passo que outros afirmam que no geral os custos seriam semelhantes e até mesmo menores em relação à $\mathrm{HTA}^{9,10}$.

O presente estudo tem como objetivo comparar os custos hospitalares entre a HVAL e a HTA, relatando a experiência inicial com a nova técnica colocada em prática em um hospital geral comunitário de médio porte.

\section{Pacientes e Métodos}

Foi realizado um estudo retrospectivo, longitudinal, não experimental, comparando os casos de HVAL e os de HTA, realizados pelo mesmo cirurgião no período de 01 de setembro de 1998 a 31 de julho de 1999, na Santa Casa de Misericórdia de Monte Alto - SP.

Os prontuários e os demonstrativos das despesas hospitalares de cada paciente foram revistos a fim de se coletarem as variáveis estudadas: idade, paridade, antecedente de cirurgia abdominal, indicação cirúrgica, cirurgia realizada, tempo cirúrgico, peso da peça operatória, tempo de internação, tipo de anestesia e custos hospitalares, subdivididos em gastos de centro cirúrgico e de enfermaria.

Todas as HVAL foram realizadas sob anestesia geral, utilizando instrumental cirúrgico não-descartável e eletrocoagulação para obtenção de hemostasia. As pacientes eram colocadas em posição de dorsolitotomia modificada, com ambos os joelhos ao nível da crista ilíaca, pernas abduzidas e semifletidas. Utilizou-se um manipulador uterino para adequada apresentação do órgão e seus anexos durante o ato operatório. Foi feita sondagem vesical de demora com sonda de Foley $\mathrm{n}-14$. A equipe cirúrgica era composta de três médicos: o cirurgião à esquerda da paciente, o primeiro auxiliar à direita e o segundo sentado entre as pernas da paciente, ficando responsável pela mobilização uterina.

O procedimento laparoscópico iniciou-se com a realização de pneumoperitônio de $\mathrm{CO}_{2}$ com agulha de Veress até um volume médio de 3 litros, não excedendo uma pressão máxima de $15 \mathrm{mmHg}$, e posterior inserção de trocarte de $11 \mathrm{~mm}$ por incisão intra-umbilical inferior, para passagem da óptica de visão frontal $\left(0^{\circ}\right)$. Sistematicamente foram utilizadas duas punções suprapúbicas de $5 \mathrm{~mm}$, laterais aos vasos epigástricos e ao nivel das cristas iliacas, sob visão direta. Uma terceira punção suprapúbica de $5 \mathrm{~mm}$, realizada na linha mediana, foi utilizada em apenas um caso. Seguiu-se cuidadosa inspeção abdominal e pélvica, com especial atenção para os anexos uterinos, ureteres e fundo de saco posterior, com eventual lise de aderências. Coagulação com pinça bipolar e corte dos ligamentos redondos. Também por coagulação bipolar e corte era efetuada a liberação anexial (ligamento infundíbulo-pélvico ou ligamento úteroovárico e trompa, conforme necessidade de anexectomia simultânea ou não). A prega peritoneal anterior era cauterizada e seccionada, seguida de dissecção do espaço vésico-uterino por meio de tesoura laparoscópica. Posteriormente à dissecção ampla dos ligamentos largos e exposição das artérias uterinas, as mesmas eram coaguladas e seccionadas conforme conveniência. O mesmo procedimento era aplicado aos ligamentos uterossacros, passando-se para o tempo vaginal.

Incisão circular na cúpula vaginal, iniciando-se pela colpotomia anterior; pinçamento, secção e ligadura dos ligamentos cardinais (Mackenrodt); ligadura dos vasos uterinos (nos casos em que o pedículo uterino não havia sido tratado por via laparoscópica) e retirada da peça. Finalmente sutura transversal da cúpula vaginal com fio absorvivel, reparando-se os ligamentos cardinais em cada ângulo para pexia, e englobando-se na sutura os ligamentos uterossacros.

Sistematicamente foi utilizado um tempo laparoscópico final para remover eventuais coágulos e assegurar a hemostasia.

As HTA foram realizadas pelas técnicas habituais, que se encontram descritas em múltiplas referências, e, como já mencionado, sempre pelo mesmo cirurgião, formando assim um grupo bastante homogêneo e passivel de comparação com as HVAL.

A análise estatística dos resultados foi feita com auxilio do programa InStat ${ }^{\circledR}$ versão 3.01 da GraphPad Software Inc. Utilizou-se o teste $\mathrm{t}$ de Student não-pareado (com correção de Welch para os casos de desvio padrão com diferença estatisticamente significativa) para a análise das variáveis quantitativas contínuas de distribuição normal, o teste de Mann-Whitney para a análise das variáveis quantitativas de distribuição livre (nãoparamétrica) e o teste exato de Fisher para a análise das tabelas de contingência, sendo $\mathrm{p}$ considerado estatisticamente significativo quando menor que $0,05(p<0,05)$.

\section{Resultados}

Onze histerectomias vaginais assistidas por laparoscopia e 23 histerectomias totais abdominais foram realizadas no periodo de estudo. Nenhuma das pacientes apresentou complicações anestésicas 
e no grupo das HVAL não houve necessidade de conversão em laparotomia em nenhum dos casos.

Não houve diferença estatística entre os grupos quanto à idade, paridade e antecedentes de cirurgia abdominal. A idade média foi de 43,6 anos $(32,5$ a 64,2$)$ e de 46,2 anos $(30,1$ a 68,0$)$ para as HVAL e HTA, respectivamente. Quanto à paridade, não houve diferença estatística $(p=0,08)$, cabendo salientar que no grupo das HVAL a proporção de pacientes com 3 ou mais filhos foi menor que para as HTA, com 18,2 e 47,8\%, respectivamente $(p=0,02)$. Mesmo nos casos de paridade elevada não havia pacientes com prolapso uterino. $\mathrm{O}$ antecedente de cirurgia abdominal prévia mostrou-se bastante homogêneo entre os dois grupos, 63,4 e $73,9 \%$ para as HVAL e HTA, respectivamente, sendo que em ambos os principais antecedentes caracterizaram-se por cesariana e laqueadura tubária. Em apenas uma paciente de cada grupo havia antecedente de cirurgia anexial, excluindo-se a esterilização cirúrgica.

Tabela 1 - Indicação para a cirurgia

\begin{tabular}{lcc}
\hline Indicação & HTA & HVAL \\
\hline Leiomiomatose uterina & 17 & 5 \\
Com algia pélvica & 7 & 4 \\
Com sangramento anormal & 4 & 0 \\
Sem dor e sem sangramento & 6 & 1 \\
Afeção endometrial $^{*}$ & 3 & 2 \\
Hemorragia uterina disfuncional $_{\text {Algia pélvica crônica** }}^{*}$ & 2 & 1 \\
Massa anexial $^{\text {Com algia pélvica }}$ & 0 & 2 \\
Sem algia pélvica & 1 & 1 \\
\hline
\end{tabular}

* Incluindo: hiperplasias endometriais e pólipo endometrial (sempre com sangramento anormal associado)

${ }^{* *}$ Incluindo: endometriose, aderências e dor de origem não esclarecida

As indicações cirúrgicas estão resumidas na Tabela 1. A principal indicação cirúrgica em ambos os grupos foi leiomiomatose uterina, correspondendo a 73,9 e $45,5 \%$ nos grupos das HTA e HVAL, respectivamente $(\mathrm{p}=0,14)$. Todos casos de afecção endometrial e de hemorragia caracterizaram-se por falha nos tratamentos prévios em controlar o sangramento uterino anormal. Quanto aos dois casos de massa anexial, o exame anátomopatológico revelou cistoadenoma seroso do ovário no caso do grupo das HTA e cisto de inclusão peritoneal (pseudocisto peritoneal) no das HVAL. Apenas uma paciente foi operada com diagnóstico de endometriose, sendo que a mesma também apresentava extenso quadro de aderências pélvicas e endometrioma do ovário esquerdo. Houve um maior número de casos de algia pélvica associado à indicação cirúrgica no grupo das HVAL (7/11 das pacientes) em relação ao grupo das HTA (7/17 das pacientes), porém essa diferença não se mostrou estatisticamente significativa $(p=0,13)$.

Tabela 2 - Variáveis intra e pós-operatórias

\begin{tabular}{lccc}
\hline Variável & HVAL & HTA & p \\
\hline Cirurgia - Duração (min) & $162,3 \pm 42,0$ & $106,3 \pm 20,5$ & $<0,01$ \\
Anestesia geral & $100,0 \%$ & $17,4 \%$ & $<0,01$ \\
Peça - Peso (g) & $125,0 \pm 42,9$ & $233,3 \pm 192,5$ & $<0,05$ \\
Diárias (dias) & 1 (1 a 2) & $2(1 \mathrm{a} 4)$ & $<0,01$ \\
Total de Gastos (R\$) & $514,53 \pm 65,51$ & $366,97 \pm 54,91$ & $<0,01$ \\
$\quad$ Centro Cirúrgico & $410,66 \pm 40,09$ & $212,46 \pm 43,55$ & $<0,01$ \\
Enfermaria & $103,87 \pm 39,26$ & $154,51 \pm 42,36$ & $<0,01$ \\
\hline
\end{tabular}

A Tabela 2 mostra os resultados das variáveis intra e pós-operatórias. Os tipos de anestesia utilizados nas pacientes submetidas à HTA foram: 15 casos de raquianestesia isolada, três de raquianestesia e peridural associadas, dois de raquianestesia e anestesia geral combinadas (endovenosa e / ou inalatória), dois de peridural e anestesia geral combinadas (endovenosa e / ou inalatória) e um de peridural isolada. No grupo das HVAL houve apenas um caso de peridural associada à anestesia geral, sendo os demais de anestesia geral isolada.

A comparação do peso das peças operatórias mostrou diferença estatística, indicando que as HVAL removeram úteros de menor volume (com pesos variando entre 50 e $220 \mathrm{~g}$ ); este menor volume uterino foi atribuído ao maior número de indicações por algia pélvica crônica associado às HVAL e a um caso de útero atrófico com pólipo endometrial e sangramento pós-menopausa.

Apenas as cinco primeiras pacientes do grupo das HVAL permaneceram internadas por dois dias, sendo que as demais tiveram alta hospitalar no dia seguinte ao procedimento; já para o grupo das HTA, em apenas um caso houve alta hospitalar no primeiro dia pós-operatório $(\mathrm{p}<0,01)$.

As HVAL mostraram-se $40,2 \%$ mais caras que as HTA. Os gastos de centro cirúrgico contribuíram com a maior parte dos custos hospitalares em ambos os grupos, correspondendo a 79,8 e $57,9 \%$ do total, para as HVAL e HTA, respectivamente. Os gastos de enfermaria para as HVAL foram menores que para as HTA, com diferença estatisticamente significativa $(\mathrm{p}=0,002)$. As Figuras 1 e 2 mostram os gastos em ordem cronológica por paciente, demonstrando que durante o período de estudo os custos relacionados às HVAL apresentaram queda, ao passo que para as HTA os mesmos se mostraram praticamente constantes (ver as curvas de tendência linear dos gráficos). 


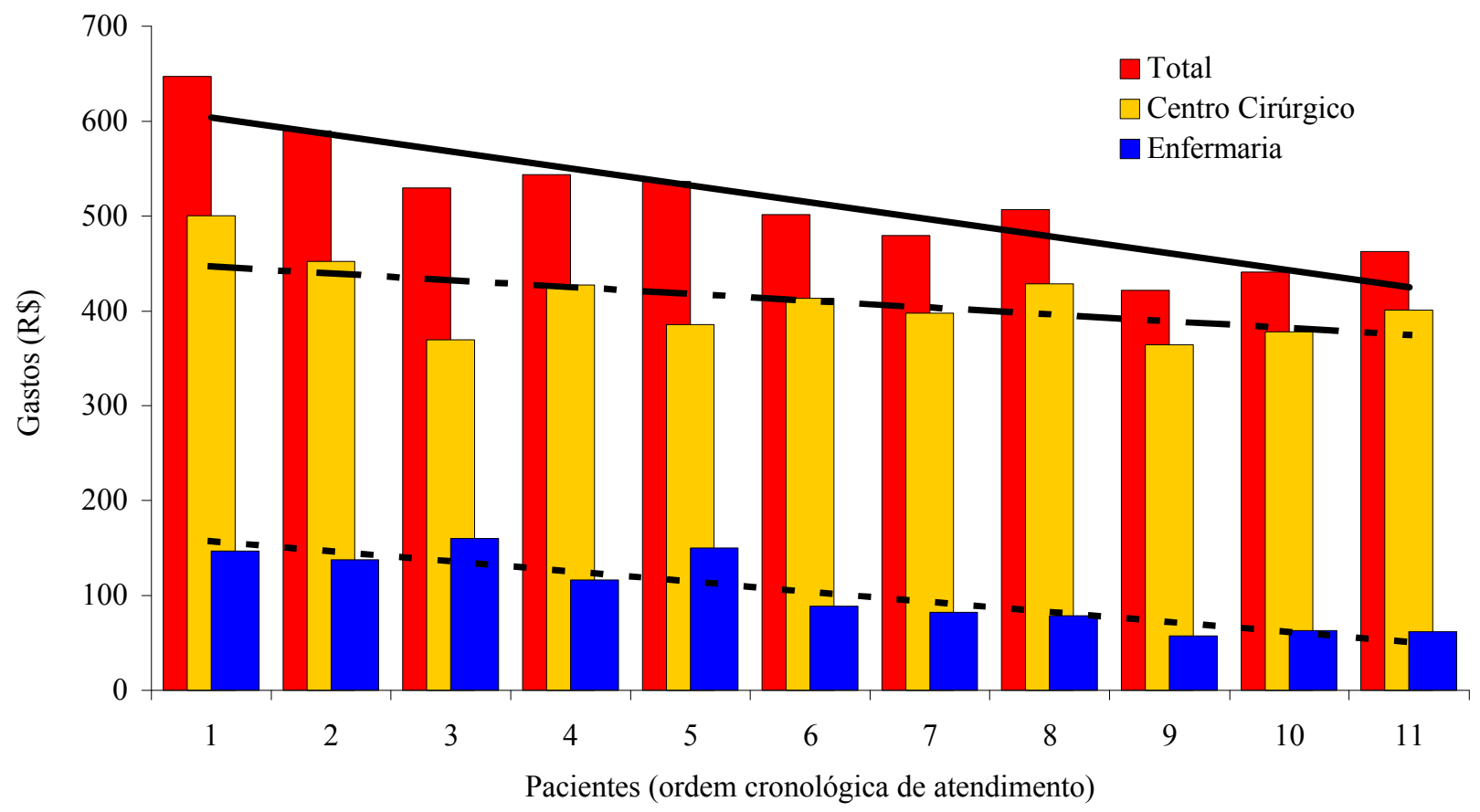

Figura 1 - Gastos com pacientes submetidas à histerectomia vaginal assistida por laparoscopia em reais

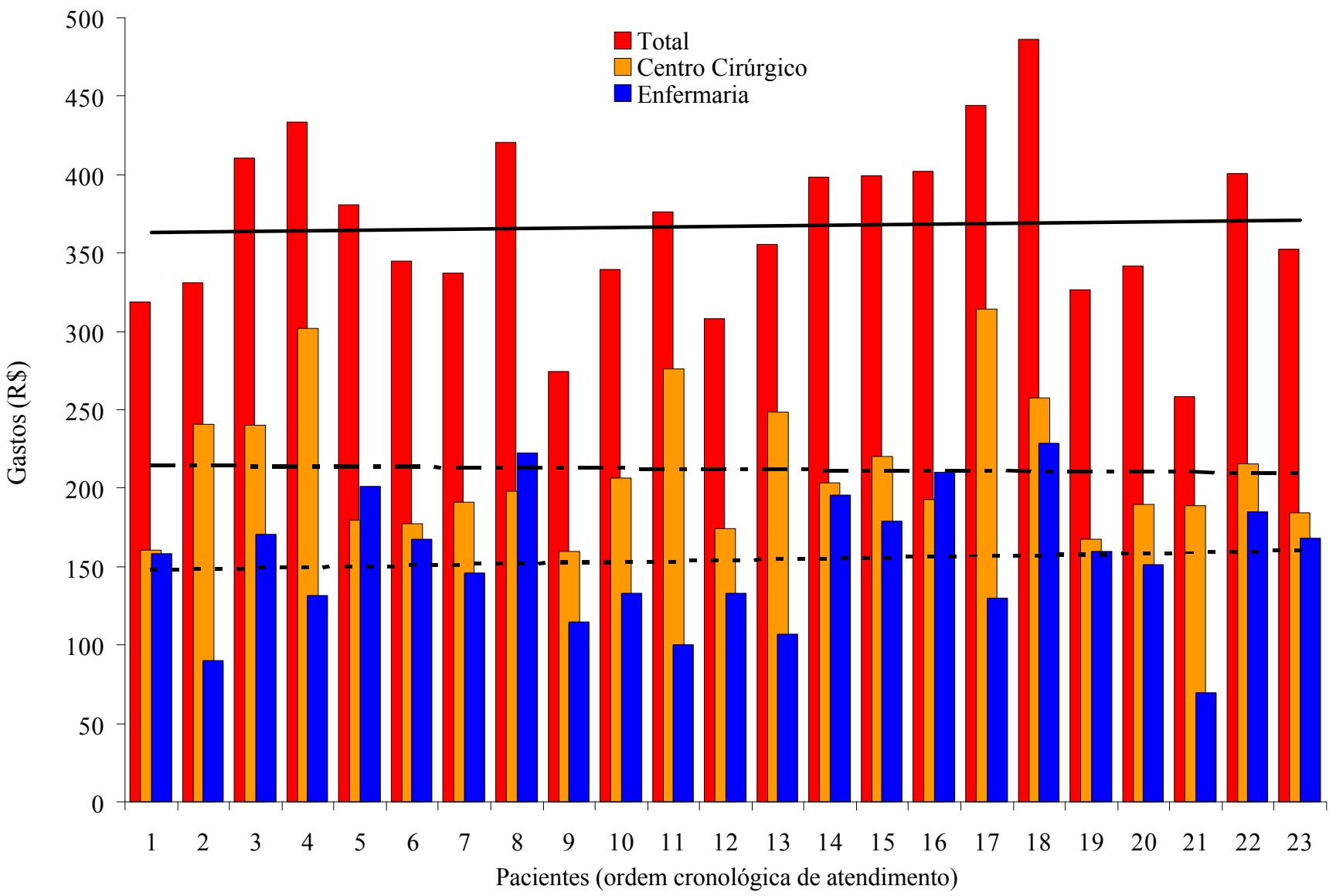

Figura 2 - Gastos com pacientes submetidas à histerectomia total abdominal em reais 


\section{Discussão}

As pacientes submetidas à HVAL têm menor desconforto e dor no período pós-operatório, período de internação mais curto e recuperação mais rápida que as submetidas à $\mathrm{HTA}^{11,12}$. O presente estudo confirma estas vantagens para a HVAL, apesar de não ter se voltado para a análise direta das variáveis de condições pós-operatórias e recuperação, pois o fato dos custos de enfermaria terem sido menores para as HVAL indiretamente traduzem esta ocorrência. O estudo refere-se à experiência inicial do cirurgião com a nova técnica, sendo que nos primeiros casos as prescrições e condutas de pós-operatório assemelhavam-se muito às das HTA. Com o passar do tempo, notou-se rapidamente a menor necessidade de analgésicos, possibilidade de re-introdução mais breve de dieta, menor necessidade de hidratação e medicamentos por via parenteral, resultando nas altas precoces e nos menores gastos de enfermaria.

A HVAL tem se mostrado um procedimento mais demorado que as outras modalidades de histerectomias. Estudos utilizando hemostasia por diatermia bipolar e instrumental não-descartável relatam diferentes tempos para a realização das HVAL. Enquanto Reis et al. ${ }^{13}$ apresentaram resultados muito semelhantes aos nossos (163,9 min, contra $162,3 \mathrm{~min}$ em nossa casuística), outros autores em suas experiências iniciais obtiveram valores bastante superiores: Daniell et al. ${ }^{14} \mathrm{com}$ $223 \mathrm{~min}$ e Torres del Toro et al. ${ }^{15}$ com $271 \mathrm{~min}$. Séries com maior número de casos, porém, mencionam tempos cirúrgicos bastante inferiores. Por exemplo, Johns e Diamond ${ }^{16}$, em casuística de 119 pacientes, relatam como tempo médio para o procedimento $79 \mathrm{~min}$. Os cirurgiões ginecológicos que estiverem começando a utilizar as técnicas laparoscópicas para a realização de histerectomias devem manter em mente que, passada a curva de aprendizado, se obtém tempos cirúrgicos cada vez mais curtos. O tempo necessário para a realização de um procedimento cirúrgico não se relaciona diretamente com a sua eficácia. As técnicas laparoscópicas têm se demonstrado bastante seguras na realização de histerectomias ${ }^{17}$, apesar de sua maior duração. Por outro lado, foi mostrado que a maior parte dos custos hospitalares se deram no centro cirúrgico, sendo intuitivo que com a queda dos tempos operatórios haja também uma redução das despesas.

Todos os estudos comparativos da revisão bibliográfica apresentada, assim como o nosso, apontam que pela via abdominal são retiradas peças cirúrgicas de maior peso ${ }^{2,4,7,9,11}$. Encontramos estudos com resultados semelhantes aos obtidos em nossa casuística de HVAL. Johns et al. ${ }^{9}$ obtiveram como peso médio $129 \mathrm{~g}$ em 839 casos e Sultana e Sogor ${ }^{2}, 123$ g em 10 casos. Em semelhança aos nossos resultados, alguns trabalhos também tiveram em comum um número considerável de indicações cirúrgicas por algia pélvica; Langebrekke et al. ${ }^{18}$ relatam como peso médio 145 g e 4/10 pacientes com dismenorréia como indicação cirúrgica; Johns e Diamond ${ }^{16}$ relatam 138 g e 67/119 pacientes com dor pélvica, e Torres del Toro et al. ${ }^{15}$ relatam $101 \mathrm{~g}$ e $7 / 12$ pacientes com adenomiose e/ou endometriose. É importante ressaltar que a HVAL não se limita a remoção de úteros pequenos, sendo que Chapron et al. ${ }^{17}$ apresentam pesos consideravelmente mais elevados, com média de 258,1 g e casos chegando a $860 \mathrm{~g}$, por exemplo.

A existência de uma massa anexial e a necessidade obrigatória da realização de ooforectomia simultânea são fatores que habitualmente fazem com que os cirurgiões ginecológicos optem pela via abdominal na realização de uma histerectomia. Este fato demonstra que há espaço para as técnicas laparoscópicas, pois estas permitem facilmente a remoção dos ovários, com abordagem direta e segura do ligamento infundíbulo-pélvico, oferecendo assim vantagem também sobre a via vaginal exclusiva $^{13,19}$. A abordagem laparoscópica tem sido utilizada inclusive em casos de câncer de endométrio quando, além da anexectomia bilateral, se faz necessário adequado estadiamento da neoplasia e linfadenectomia pélvica ${ }^{20,21}$, sendo que anteriormente não havia outra opção que não fosse a via abdominal.

A comparação de custos hospitalares entre trabalhos da literatura torna-se muito complicada por várias razões: diferenças econômicas e cambiais entre os países; forma de computação dos custos e suas subdivisões; padrões diferentes de internação quanto à hotelaria hospitalar; treinamento prévio e capacitação profissional com as técnicas laparoscópicas; associação de outros procedimentos (reparo do colo vesical, correções de paredes vaginais e apendicectomia, por exemplo); utilização de instrumentais descartáveis, grampeadores e laser, dentre outras. Devido a tantas diferenças, apenas os estudos que enfocam custos entre os diferentes tipos de histerectomia têm algum valor, respeitando-se neste caso a semelhança entre os grupos e a igualdade de condições.

Van den Eeden et al. ${ }^{10}$ demonstraram custos gerais menores para o grupo das HVAL em relação às HTA, porém também obtiveram gastos mais elevados de centro cirúrgico que em seu estudo foram suplantados quando computados gas- 
tos de enfermaria. Nossa casuística provém de um hospital beneficente e sem fins lucrativos, com valores baixos de diárias e taxas, e talvez por este motivo, mesmo com custos pós-operatórios significativamente menores, não conseguimos a mesma inversão. Nossas pacientes foram internadas em enfermarias com custo diário de $\mathrm{R} \$ 25,00$ (por sistema de convênio local); foram um total de 16 diárias para as HVAL e 55 para as HTA, correspondendo a 7,1 e 16,3\% do total de custos de cada grupo respectivamente. Considerando-se que este preço não representa a realidade da maioria das instituições e que os custos das HVAL apresentaram queda, pode-se concluir que sendo o mesmo levantamento feito em outras condições de internação e passada a curva de adaptação à nova técnica, o resultado poderia ser semelhante ou mesmo contrário ao observado nesta casuística. Nossas HVAL mostraram-se 40,2\% mais caras, porém os valores das HTA apresentados também são bastante baixos, uma vez que foram levantados no mesmo hospital e sob o mesmo regime de internação. Por estas razões, não consideramos que o custo médio de $\mathrm{R} \$ 515,00$ seja proibitivo para a realização de uma cirurgia laparoscópica classificada como avançada.

Levando-se em conta que esta é apenas nossa experiência inicial com a utilização das técnicas laparoscópicas para a realização de histerectomias, os tempos cirúrgicos e as despesas que tendem a cair, as vantagens oferecidas já mencionadas e preços não-proibitivos, concluímos que a HVAL, quando executada em um hospital geral de médio porte, apesar de mais cara que a HTA, representa uma ótima opção para a remoção uterina e deve fazer parte do arsenal terapêutico dos cirurgiões ginecológicos.

\section{SUMMARY}

Purpose: to compare hospital costs between laparoscopically assisted vaginal hysterectomy (LAVH) and total abdominal hysterectomy (TAH), reporting the initial experience with the new approach in a communitary general hospital.

Patients and Methods: eleven cases of LAVH and 23 of TAH, carried out from September 1998 to July 1999, were compared. Each patient's records and hospital charges were reviewed to collect the analyzed variables.

Results: there was no statistical difference between the groups in relation to age, parity, and previous abdominal surgery. The main surgical indication for both groups was uterine leiomyomatosis. The LAVH group presented a shorter hospital stay with a median of one day, and the TAH group, of two days $(p<0.01)$. LAVH showed to be $40.2 \%$ more expensive than TAH $(p<0.01)$. Operating room charges contributed to the major part of hospital costs for both groups, corresponding to 79.8 and $57.9 \%$ of the total, for LAVH and TAH, respectively. LAVH infirmary charges were smaller than for TAH, with a statistically significant difference $(p=0.002)$.

Conclusion: with shorter hospital stay and smaller infirmary costs, we demonstrated that LAVH provides better postoperative conditions and faster recovery than TAH. When done in a community general hospital, despite being more expensive, LAVH is an excellent option for uterine removal, and should be part of the therapeutical arsenal of gynecologic surgeons.

KEY WORDS: Hysterectomy. Laparoscopy. Health care costs.

\section{Referências}

1. Reich H, DeCaprio J, McGlynn F. Laparoscopic hysterectomy. J Gynecol Surg 1989;5: 213-6.

2. Sultana CJ, Sogor L. Laparoscopy vs. laparotomy for gynecologic procedures. Impact on resident training. J Reprod Med 1996;41: 225-30.

3. Richardson RE, Bournas N, Magos, AL. Is laparoscopic hysterectomy a waste of time? Lancet 1995;345: 36-41.

4. Kably Ambe A, Barroso Villa G, Jurado Jurado M, Almanza R, García León F. Histerectomía vaginal asistida por laparoscopia. Estudio crítico y comparativo con histerectomia vaginal y abdominal en el Hospital A.B.C. de la Ciudad de México. Ginecol Obstet Mex 1997;65: 362-7.

5. Minelli L, Angiolillo M, Caione C, Palmara V. Laparoscopically-assisted vaginal hysterectomy. Endoscopy 1991;23: 64-6.

6. Chapron C, Dubuisson JB. Hystérectomie totale pour pathologies bénignes: Techniques coeliochirurgicales et indications. In: Encyclopédie Médico-Chirurgicale. Techniques chirurgicales Urologie-Gynécologie. Paris: Elsevier;1995. p.12.

7. Boike GM, Elfstrand EP, DelPriore G, Schumock D, Holley HS, Lurain JR. Laparoscopically assisted vaginal hysterectomy in a university hospital: report of 82 cases and comparison with abdominal and vaginal hysterectomy. Am J Obstet Gynecol 1993;168: 1690-701.

8. Nezhat C, Bess O, Admon D, Nezhat CH, Nezhat F. Hospital cost comparison between abdominal, vaginal, and laparoscopy-assisted vaginal hysterectomies. Obstet Gynecol 1994;83: 7136.

9. Johns DA, Carrera B, Jones J, DeLeon F, Vincent R, Safely C. The medical and economic impact of laparoscopically assisted vaginal hysterctomy in a large, metropolitan, not-for-profit hospital. Am J Obstet Gynecol 1995;172: 1709-19. 
10.Van den Eeden SK, Glasser M, Mathias SD, Colwell HH, Pasta DJ, Kunz K. Quality of life, health care utilization, and costs among women undergoing hysterectomy in a managed-care setting. Am J Obstet Gynecol 1998;178: 91-100.

11. Nezhat F, Nezhat C, Gordon S, Wilkins E. Laparoscopic versus abdominal hysterectomy. J Reprod Med 1992;37: 247-50.

12.Liu CY. Laparoscopic hysterectomy. A review of 72 cases. J Reprod Med 1992; 37:351-4.

13.Reis FJC, Nogueira AA, Andrade JM, Carrara HHA, Reis PAS, Bighetti S. Histerectomia vaginal assistida por laparoscopia em pacientes com necessidade de anexectomia. Rev Bras Ginecol Obstet 1998;20: 571-6.

14.Daniell JF, Kurtz BR, McTavish G, Gurley LD, Shearer RA, Chambers JF, et al. Laparoscopically assisted vaginal hysterectomy. The initial Nashville, Tennessee, experience. J Reprod Med 1993;38: 537-42.

15.Torres del Toro B, Peimbert M, Torres JM, Santos R, Romero L. Histerectomía laparoscópica (HVAL). Experiencia inicial. Ginecol Obstet Mex 1994;61: 1-6.
16.Johns DA, Diamond MP. Laparoscopically assisted vaginal hysterectomy. J Reprod Med 1994;39: 424-8.

17.Chapron CM, Dubuisson JB, Ansquer Y. Is total laparoscopic hysterectomy a safe surgical procedure? Hum Reprod 1996;11: 2422-4.

18.Langebrekke A, Skår OJ, Urnes A. Laparoscopic hysterectomy. Initial experience. Acta Obstet Gynecol Scand 1992;71: 226-9.

19.Phipps JH, John M, Nayak S. Comparison of laparoscopically assisted vaginal hysterectomy and bilateral salpingo-oophorectomy with conventional abdominal hysterectomy and bilateral salpingo-oophorectomy. $\mathrm{Br} \mathrm{J}$ Obstet Gynaecol 1993;100: 698-700.

20.Spirtos NM, Schlaerth JB, Gross GM, Spirtos TW, Schlaerth AC, Ballon SC. Cost and quality-oflife analyses of surgery for early endometrial cancer: laparotomy versus laparoscopy. Am J Obstet Gynecol 1996;174: 1795-800.

21.Barbosa CP, Moscovitz T, Martins NV, Souza AMB, Soubhia IT, Aldrighi APS. Abordagem laparoscópica do câncer de endométrio. Rev Bras Ginecol Obstet 1999;21: 41-5.

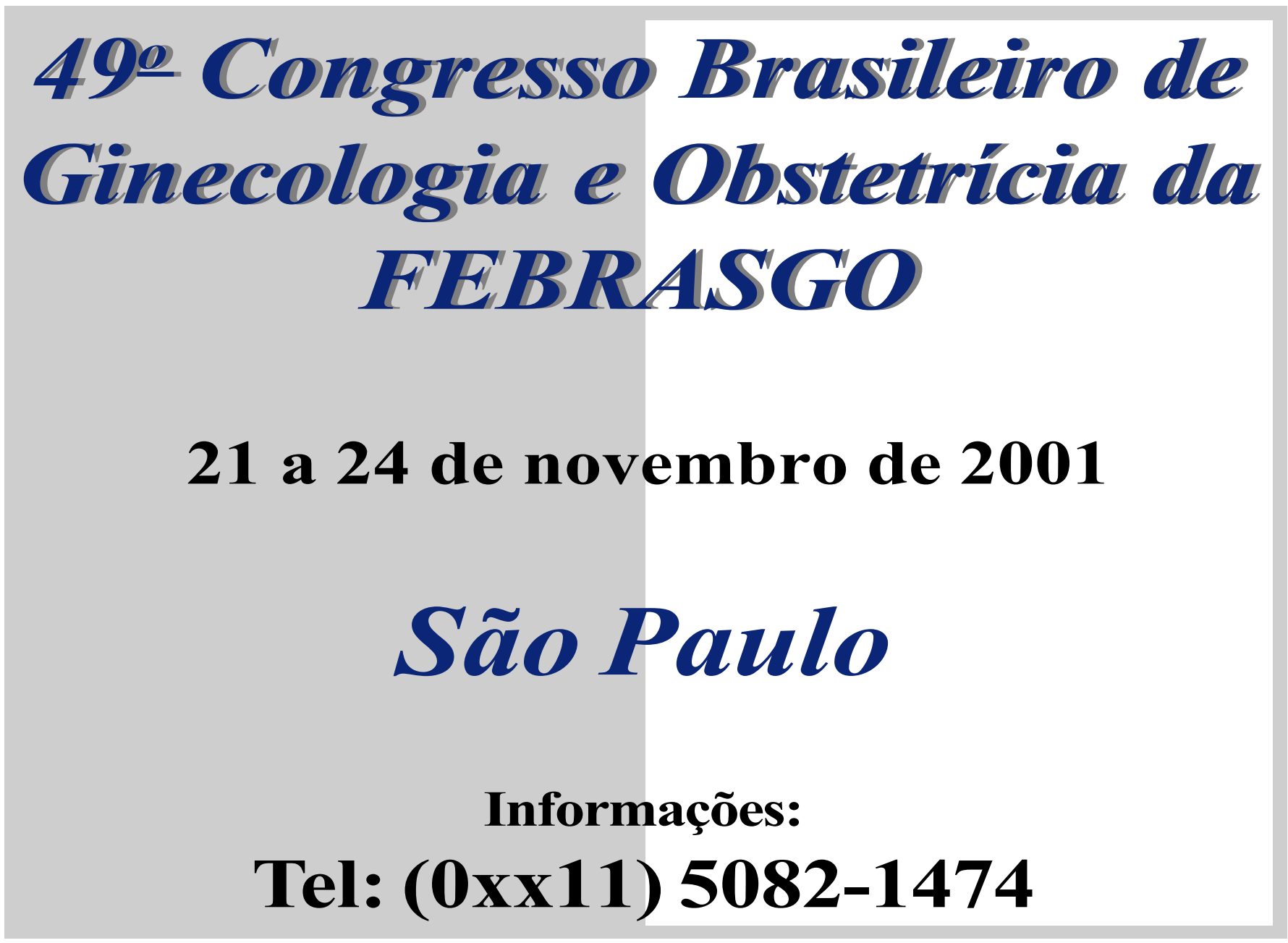

